\title{
INVESTIGATING EFFECT OF NURSING INTERVENTIONS, BASED ON WELLS SCORE RESULTS, ON THE INCIDENCE OF DEEP VEIN THROMBOSIS IN PATIENTS ADMITTED TO THE INTENSIVE CARE UNIT
}

\author{
MORTEZA HABIBI MOGHADAM ${ }^{1}$, MARZIEH ASADIZAKER ${ }^{1}$, SIMIN JAHANI ${ }^{2}$, ELHAM MARAGHI*, \\ HAKIMEH SAADATIFAR ${ }^{4}$, FARHAD NAANAEI ${ }^{5}$
}

${ }^{1}$ Nursing Care Research Center in Chronic Diseases, School of Nursing and Midwifery, Ahvaz Jundishapur University of Medical Sciences, Ahvaz, Iran. ${ }^{2}$ Nursing Care Research Center in Chronic Diseases, Ahvaz Jundishapur University of Medical Sciences, Ahvaz, Iran. ${ }^{3}$ Department of Biostatistics and Epidemiology, School of Public Health, Ahvaz Jundishapour University of Medical Sciences, Ahvaz, Iran. ${ }^{4}$ Department of Echocardiography, Dezful University of Medical Sciences, Dezful, Iran. ${ }^{5}$ Department of Anesthesiology, Dezful University of Medical Sciences, Dezful, Iran. Email: marziyehasadizaker@gmail.com

Received: 25 January 2018, Revised and Accepted: 14 February 2018

ABSTRACT

Objective: Venous thromboembolism, including deep vein thrombosis (DVT) and pulmonary embolism (PE), is a common complaint in critically ill patients. Therefore, the present study was conducted to determine the effect of nursing interventions, based on the Wells results, on the incidence of DVT in intensive care unit (ICU) patients.

Methods: The present clinical trial was conducted on 72 ICU patients without DVT and PE who met the inclusion criteria according to Wells score in Dr. Ganjavian Hospital, Dezful in 2017. The participants were investigated and randomly divided into intervention $(n=36)$ and control groups $(n=36)$. The intervention group received preventive nursing measures based on the risk level determined by the Wells score, and routine therapeutic interventions were performed for the control group. Then, patients were evaluated using Wells score, D-dimer testing, and Doppler sonography on the $1^{\text {st }}, 5^{\text {th }}$, and $10^{\text {th }}$ days. Data were finally coded and entered into SPSS version 23 . Data analysis was performed using Chi-square, Fisher's exact, and Mann-Whitney U tests.

Results: The incidence of DVT in both groups showed that 2 patients of the control group who were identified to be at risk using the Wells score were diagnosed with DVT while none of the patients of the intervention group experienced DVT. The present study showed that $22.2 \%$ of the patients of the control group suffered from non-pitting edema, which was significantly different from the intervention group ( $\mathrm{p}=0.005)$.

Conclusion: The results of the present study showed that using the Wells score for early identification of the at-risk patients and nursing interventions based on this score's results is helpful in the prevention of DVT. Appropriate nursing interventions were also effective in reducing the incidence of non-pitting edema in the lower extremities.

Keywords: Deep vein thrombosis, Wells score, Nursing interventions.

(c) 2018 The Authors. Published by Innovare Academic Sciences Pvt Ltd. This is an open access article under the CC BY license (http://creativecommons. org/licenses/by/4. 0/) DOI: http://dx.doi.org/10.22159/ajpcr.2018.v11i5.24939

\section{INTRODUCTION}

Deep vein thrombosis (DVT) is one of the leading causes of death worldwide and usually occurs in the lower extremity and is associated with life-threatening consequences, pulmonary embolism (PE), and is known as venous thromboembolism (VTE) [1]. VTE is a common complaint in critically ill patients [2]. These patients are prone to DVT due to factors such as need for surgery, catheter, immobility, and use of sedatives or neuromuscular-blocking drugs [3]. The exact global number of individuals with VTE is still unknown. According to the latest Centers for Disease Control and Prevention data statistics released in June 2015, a total of 900,000 individuals are afflicted with VTE (in the United States, 1-2 out of 1000 patients) per year. Of these, about 60,000 to 100,000 individuals die, 1-s of them are infected with longterm complications (post-thrombotic syndrome), and one-third of them experience recurrence within 10 years [1]. The findings of Shakur et al.' study which was conducted in Iran indicated that the frequency percentage of lower extremity DVT was $53.6 \%, 46.3 \%, 45.3 \%$, and $48.5 \%$ in the surgical intensive care unit (ICU), respiratory ICU, neuroICU and for the total patients, respectively [3]. Furthermore, according to Safavi et al's study that was conducted in Tehran, the incidence of VTE was $9 / 10000$ and the PE-related mortality rate was $11 \%$ in hospitalized patients [4]. Rezaei et al. showed that the majority of ICU patients are at risk of developing DVT [5]. Many patients with DVT lack clinical symptoms and these patients are identified when they have PE [4] while PE has been shown to be the most common cause of undiagnosed death in hospitalized patients [6]. Hence, ICU nurses play a key role in preventing DVT and its complications. They are in an ideal position to assess early risk factors for the patient and to take appropriate measures to prevent DVT [7]. Collins et al. [8] demonstrated in a study that using a coherent nursing program on 2063 hospitalized patients over a period of 5 years resulted in a decrease among patients at the risk of DVT from 27\% in 2005 to $85 \%$ decrease in 2009. Therefore, nurses responsible for risk assessment and DVT prevention can significantly reduce its incidence and subsequent complications [8]. Clinical stages of DVT can be very dangerous, so it is of vital importance to detect it early in high-risk patients [3]. Hence, numerous scoring systems, such as Hamilton score, AMUSE score, and Wells clinical prediction rule, are available to determine the pre-test probability of DVT. At the meantime, the Wells score is probably better known than others. DVT Wells score has been widely studied and recommended as a first step in the diagnostic algorithm to pre-test the risk assessment in suspected DVT patients [9]. It can also be used for outpatients or inpatients [10]. Based on Wells clinical prediction rule (1997), patients were divided into low-, moderate-, and high-risk groups with DVT prevalence rate of $5 \%, 17 \%$, and $53 \%$, respectively [9]. However, according to the modified Wells 
model (2003) which has score range of 2-9, patients are divided into two groups with the score of $<2$ and $>2$ indicates the presence and absence of the risk of DVT development, respectively [11]. Experts suggest using modified Wells two-level pre-test for its simplicity [10]. Previous studies have reported that most health professionals perform DVT risk assessment based on their experience, which is not suitable for clinical evaluation of patients [12]. For example, in response to a standard questionnaire sent to all 394 physicians in the Italian Association for Thrombosis and Hemostasis, 22\% of physicians acknowledged that they had never used a clinical guideline for patients with a suspected DVT [13]. Hence, studies recommend that health professionals should be more focused on DVT prevention and identify at-risk patients using an effective DVT assessment tool so that the mortality rate is reduced effectively by implementing appropriate measures [14]. Now, considering the lack of continuous access to the physician, on the one hand, and the continuous presence of nurses on the patients' bedside on the other hand, nursing care interventions play an important in preventing the development of embolism and its timely diagnosis [15]. Considering the importance of the role and position of nursing in evaluating the risk of VTE [8], as well as the use of Wells modified two-level pre-test for patients recommended by experts [10], the present aimed to determine the effect of nursing interventions on incidence of DVT in ICU patients based on the Wells modified score.

\section{METHODS}

This study is a randomized clinical trial designed to determine the effect of nursing interventions, based on Wells score results, on incidence of DVT in ICU patients who referred to Dr. Ganjavian Hospital, Dezful, Iran, in 2017. The present study was approved by the Ethics Committee of Ahvaz Jundishapur University of Medical Sciences with the code of ethics: IR.AJUMS.REC.1396.378 and the clinical trial code: IRCT20170624034723N1. Patients with trauma and patients with brain lesions who were diagnosed as having no DVT and PE were enrolled in the present study. The inclusion criteria included the age range of 18-65 and the minimum length of stay of 10 days. Pregnant women, patients with hemodynamic disorders, patients with coronary artery disease at the acute phase, a diagnosis of imminent death by the physician, and lack of cooperation of the patient or his/her supervisor were among the exclusion criteria of the present study. Before being admitted, patients submitted their written informed consent. The data were collected by the researchers and recorded in the checklist designed for this purpose. Necessary and routine treatments were performed for all patients based on the ethical issues. The data include demographic characteristics (age, gender, marital status, employment, education, body mass index [BMI]), underlying diseases (diabetes, hypertension, and obesity), history of smoking, medication history, history of DVT, bed rest status, diet, disease diagnosis, results of Wells score, D-dimer testing, and Doppler ultrasound test. After meeting the inclusion criteria, the patients were evaluated according to the modified Wells tool and randomly assigned to intervention $(n=36)$ and control groups $(n=36)$. Patients were divided into two levels of with and without risk of DVT in such way that patients with scores of $>2$ and $<2$ were divided into two levels of at risk and the ones with unlikely risk of DVT, respectively. The intervention was assigned randomly to patients using stratified random sampling method, and the randomized list was prepared by a statistic consultant. A person outside the study assigned patients to the intervention and control groups according to a randomized list and the corresponding codes for each patient who was enrolled in the study. After determining the risk level based on the modified Wells score, atrisk patients and the ones with unlikely risk of DVT in the control group received routine and required treatments of the ward based on their physician's instructions and according to ethical considerations. In the intervention group, patients with unlikely risk of DVT determined by Wells score received the following nursing interventions: Limb warming, elevating legs above the heart level (periodically for $20 \mathrm{~min}$ ), limb exercising (flexion and dorsiflexion of the legs, 15-20 times for each limb), respiratory, and cough exercises based on a checklist designed for three times in the morning, evening, and night. In addition to the above measures, mechanical preventive devices (anti-embolism stockings) were used for patients at risk of DVT according to the Wells score. Patients in both control and intervention groups were evaluated using D-dimer and Doppler ultrasound tests on the $1^{\text {st }}, 5^{\text {th }}$, and $10^{\text {th }}$ days based on the modified Wells score.

In a study, Mozafar et al. calculated sensitivity, specificity, positive predictive value (PPV), and negative predictive value (NPV) of Wells score in combination with D-dimer results in DVT groups. PPV value for the at-risk groups with positive D-dimer results and NPV value for low-risk groups with negative D-dimer results were $85.71 \%$ and 96\%, respectively [16]. Furthermore, the findings of Hekmatnia and Pourmoghaddas study showed that the Doppler ultrasound has sensitivity and specificity of $100 \%$ and $17 \%$ in diagnosing for femoral and popliteal vein thrombosis, respectively [17]. Finally, the collected data were analyzed using SPSS version 23, and the results were analyzed and reported using Chi-square, Fisher's exact, and MannWhitney U tests.

\section{RESULTS}

The results of Table 1 show that there was no significant difference between the two groups in terms of age and gender findings. The present study investigated a total of 72 patients with mean age of $45.83 \pm 16.06$ years in the intervention group and $45.94 \pm 14.07$ years in the control group. The male and female patients accounted for $56(77.8 \%)$ and $16(22.2 \%)$ of the participants, respectively. Furthermore, the prevalence of underlying diseases, including diabetes, hypertension, BMI and smoking history were compared in the two studied groups (Table 1), which according to the results, there was no significant difference between the two groups. The findings of Table 2 indicate that the two groups are homogeneous for diagnosis of the disease, level of mobility, and consciousness. In the present study, brain lesion (cerebrovascular accident-intracerebral hemorrhage [CVAICH]), trauma, and post-cardiopulmonary resuscitation were observed among 23,45 , and 4 patients, respectively. A total of $50(69.4 \%)$ and $22(30.6 \%)$ of patients had absolute rest (immobility) and relative rest, respectively. There were 60 patients with mild loss of consciousness (Glasgow Coma Scale [GCS]: 13-15) and 12 patients with an average loss of consciousness (GCS: 9-12). The findings of Table 3 show that the two study groups are homogeneous in terms of length of stay. Investigating the incidence of non-pitting edema of lower extremities in the two groups of the present study showed that $8(22.2 \%)$ of the patients in the control group suffered such edema in the lower extremities while no non-pitting edema was observed in the lower

Table 1: Comparison of the variables studied among the two groups of study

\begin{tabular}{llll}
\hline Variables & $\begin{array}{l}\text { Intervention } \\
\text { group (A) }\end{array}$ & $\begin{array}{l}\text { Control } \\
\text { group (B) }\end{array}$ & p-value \\
\hline $\begin{array}{l}\text { Age (year) } \\
\text { Gender (\%) }\end{array}$ & $45.83 \pm 16.06$ & $45.94 \pm 14.07$ & 0.795 \\
$\quad$ Man & $28(77.8)$ & $28(77.8)$ & $>0.99$ \\
$\quad$ Woman & $8(22 / 2)$ & $8(22 / 2)$ & \\
$\begin{array}{l}\text { BMI (\%) } \\
\quad<18.5\end{array}$ & $0(0.0)$ & $1(2.8)$ & 0.937 \\
$\quad 18.5-25$ & $16(44.4)$ & $17(47.2)$ & \\
$25-30$ & $17(47.2)$ & $15(41.7)$ & \\
$>30$ & $3(8.3)$ & $3(8.3)$ & \\
$\begin{array}{l}\text { Hypertension, } \\
\text { number (\%) }\end{array}$ & & & \\
$\quad$ Yes & $16(44 / 4)$ & $13(36 / 1)$ & 0.631 \\
$\quad$ No & $20(55 / 6)$ & $23(63 / 9)$ & \\
$\begin{array}{l}\text { Diabetes, number (\%) } \\
\quad \text { Yes }\end{array}$ & $6(16 / 7)$ & $8(22 / 2)$ & 0.767 \\
$\quad$ No & $30(83 / 3)$ & $28(77 / 8)$ & \\
Smoking, number (\%) & & & \\
$\quad$ Yes & $12(33 / 3)$ & $14(38 / 9)$ & 0.806 \\
$\quad$ No & $24(66 / 7)$ & $22(61 / 1)$ & \\
\hline
\end{tabular}

BMI: Body mass index 
extremities of the patients in the intervention group due to appropriate nursing interventions implemented based on Wells score, which was significant $(p=0.005)$. No change was made in the results of the Wells score while evaluating patients based on the Wells score on the $1^{\text {st }}, 5^{\text {th }}$, and $10^{\text {th }}$ days in such way that the risk-free patients remained in the same group, and there was no drop in the risk score of patients in the group with DVT risk and they remained in the risk group until the last day of the study. Furthermore, the results of investigating the D-dimer level of patients showed that only one of the patients in the control group had a negative D-dimer testing, which could indicate the low sensitivity of the conventional D-dimer testing for DVT diagnosis. Finally, the incidence of DVT in the two groups, which was determined based on the results of color Doppler ultrasound, showed that two patients of the control group who were identified to be at risk using a modified Wells score were diagnosed with DVT while none of the individuals in the intervention group did not experience DVT.

\section{DISCUSSION}

The aim of this study was to determine the effect of nursing interventions, based on Wells score results, on the incidence of DVT in patients who were admitted to the ICU ward due to brain lesions and head trauma. In the present study, the mean age of patients in the intervention and control groups was $45.83 \pm 16.06$ and $45.94 \pm 14.07$ years, respectively, that show both groups were homogeneous in this regard (Table 1). Moheimani and Jackson referred in their study to the age above 40 years as a risk factor for DVT incidence [11]. Furthermore, previous studies have shown that the incidence rate of VTE increases with age [10]. The results of Khavaninzadeh et al.'s study showed that there is a significant relationship between the age and the need for anti-DVT prophylaxis in such way that prophylaxis rate increases with the age [18]. According to the findings of this study, 69.4\% of patients were motionless on their bed (Table 2). The results of Kazemi et al.'s study showed that immobility is the most common cause of DVT in patients [19]. According to studies, bed rest reduces blood flow in the legs by at least 50\% [20]. The results of Hitos et al.'s study which aimed to determine the factors affecting the blood flow of popliteal veins and the most effective exercise method in the prevention of venous depression showed that blood volume flow in the popliteal vein was reduced by almost $40 \%$ with immobility of seated participants and by almost $80 \%$ when sitting motionless with feet not touching the floor [21]. The immobility of hospitalized patients often causes blood clots in the arteries. Therefore, it seems that the design and implementation of exercise programs can be effective in preventing DVT [22]. In the present study, investigating incidence of edema of lower extremities showed that $8(22.2 \%)$ of the patients in the control group had non-pitting edema in the legs while none of the patients of intervention group suffered from non-pitting edema in lower extremities (Table 3). To help improve the pain and swelling of patients with acute DVT, Elastic compression stockings should be used. Elastic compression stockings reduce accumulation of blood in the vessels of the extremities by increasing the pressure on the ankle over the upper trunk (thigh), and this can increase the rate of blood flow to the heart [11]. The results of Schwarz et al.'s study showed that none of the 115 patients using compression elastic stockings experienced leg DVT after 8-h flights [23].

It was observed in the present study that anti-embolism stockings and limb exercise were used for none of the patients of the control group while nursing interventions with limb exercise were performed for all patients in the intervention group and anti-embolism stockings were used for at-risk individuals based on the Wells score, which can justify the lack of non-pitting edema and the absence of DVT in patients of the intervention group. The results of Al-Rashedi et al.'s study showed that ICU nurses may be effective in preventing DVT through measures such as leg exercises, compression elastic stockings, deep breathing, coughing exercises, and early discharge as these measures improve venous return and reduce venous stasis in the leg veins [6]. Kwon et al.'s study aimed to investigate the effect of ankle and respiratory exercises on blood flow velocity in the femoral veins. The results showed that the blood flow rate has increased fourfold in the femoral vein after performing ankle exercise and deep breathing simultaneously, which can be used as a method to prevent DVT after orthopedic, heart, or lung surgeries [24]. Furthermore, Memariyan et al.'s study showed that the incidence of DVT decreased with the simultaneous use of compression elastic stockings and exercise. According to the results of this study, the use of two simultaneous prevention methods is more effective [25]. The results of the present study revealed that the use of the Wells score could be useful for early identification of at-risk patients. The results also showed that 2 patients in the control group who were detected as being at risk were diagnosed with DVT using Wells score, while none of the subjects in intervention group experienced DVT. Similarly, Modi et al. showed a linear relationship between the Wells score and the incidence of DVT in such way that there is no risk of developing DVT if the patient is in a low-risk group based on the Wells scoring instrument $(<1)$, and there is likelihood of DVT if (s)he is placed in the medium- or high-risk groups [26]. Dybowska et al.'s study also showed that the use of Wells score in primary care shows a high degree of accuracy [27]. However, the results of Silveira et al.'s study, which aimed to determine the function of Wells score for the evaluation of DVT in hospitalized patients, were inconsistent with the results of the present study. Accordingly, Wells score does not have the required effectiveness in hospitalized patients [28]. Wilbur and Shian also showed in their study that Wells score significantly estimates the likelihood of DVT due to the excessive overlap of clinical symptoms in patients with cellulitis [9]. In the present study, only a negative case was reported from a total of D-dimer tests performed by the laboratory of the hospital that had a typical D-dimer test, which indicates a low sensitivity of the conventional D-timer testing in DVT diagnosis. The results of the studies show that the effectiveness of D-dimer measurement is only to exclude VTE in case of the negative result, but its positive results are not diagnostic for VTE [9]. D-dimer is a degradation product of cross-linked fibrin which typically increases with acute VTE [29,30]. According to studies, D-dimer levels increase with various types of non-thrombotic disorders such as atrial fibrillation, acute coronary syndrome, stroke, severe hemorrhagic digestion, infection, disseminated intravascular coagulation and severe renal dysfunction, recent major surgery, bleeding, trauma, and malignancy as well as with age and

Table 2: Comparison of the variables studied among the two groups of study

\begin{tabular}{llc}
\hline Variables & Intervention group (A) & Control group (B) \\
\hline Diagnosis (\%) & $13(36.1)$ & $10(27.8)$ \\
$\quad$ CVA-ICH & $21(58.3)$ & $24(66.7)$ \\
HT-MT-accident & $2(5.6)$ & $2(5.6)$ \\
$\quad$ Post-CPR & $29(80.6)$ & $31(86.1)$ \\
Level of consciousness (GCS) (\%) & $7(19.4)$ & $5(13.9)$ \\
13-15 & & 0.703 \\
$9-12$ & $25(69.4)$ & $25(69.4)$ \\
Patient rest position during admission (mobility rate) (\%) & $11(30.6)$ & $11(30.6)$ \\
$\quad$ Absolute rest (CBR) & $>0.99$ \\
$\quad$ Relative rest (RBR) & \\
\hline
\end{tabular}

CVA-ICH: Cerebrovascular accident-intracerebral hemorrhage, CPR: Cardiopulmonary resuscitation, GCS: Glasgow Coma Scale, RBR: Rule-based reasoning,

CBR: Case-based reasoning, HT: Head trauma, MT: Multiple trauma 
Table 3: Comparison of length of stay and incidence of non-pitting edema in control and intervention groups

\begin{tabular}{llll}
\hline Variables & Control group & $\begin{array}{l}\text { Intervention } \\
\text { group }\end{array}$ & p-value \\
\hline $\begin{array}{l}\text { Length of stay } \\
\text { Lower extremity } \\
\text { edema }\end{array}$ & $16 / 67 \pm 14 / 89$ & $16 / 25 \pm 14 / 64$ & 0.35 \\
$\quad$ & & 0.005 \\
Yes & $8(22 / 2)$ & 0 & \\
No & $28(77 / 8)$ & $36(100)$ & \\
\hline
\end{tabular}

pregnancy $[2,29]$. Hence, D-dimer testing has a low specificity in ICU patients [2]. There were a total of 23 patients with brain lesion (CVA$\mathrm{ICH}$ ) and 45 patients with trauma in this study, which can be due to positive D-dimer in the studied patients. Therefore, studies recommend the use of the enzyme-linked immunosorbent assay (ELISA) method, a rapid quantitative ELISA, and turbidimetric D-dimer testing (advanced D-dimer) for the diagnosis of VTE, which have a sensitivity of more than 95\% [9]. Mazzolai et al. showed that a negative ELISA and D-dimer testing can eliminate DVT without further testing in $30 \%$ of patients with thromboembolic risk $<1 \%$ within 3 months after treatment [10]. One of the limitations of this research included small sample size due to lack of financial resources and time constraints. The other limitation included the problem with the discharging patients from the ICU for the color Doppler ultrasound; however, this could double the importance of using a clinical pre-test according to the triple (pre-test, D-dimmer testing, and ultrasound) recommended for DVT diagnosis by some studies. However, there is a need for further studies with larger sample size and longer length of stay.

\section{CONCLUSION}

The results of the present study showed that using the Wells score for early identification of the at-risk patients and nursing interventions based on this score's results is helpful in the prevention of DVT. Nurses can prevent DVT in ICU patients, even in the absence of a doctor, after identifying at-risk patients by using screening tools, by moving the organs of patients with effective exercise, and by using mechanical devices (anti-embolism stockings). It is recommended that all patients be evaluated in terms of the risk of developing DVT at the time of admission to the ICU using a pre-test so that we detect at-risk patients early and start preventive nursing measures, including anti-embolism stockings, exercise, and elevating legs above the heart level, begin from the first moment of admission to the ward. It is also recommended that educational programs be developed to enhance the knowledge and technical skills of physicians and nurses to assess the occurrence of DVT using screening pre-tests as well as to take preventive measures to prevent the cost of developing DVT and its complications and deaths and help promote the health status of patients and society, accordingly.

\section{ACKNOWLEDGMENTS}

This article is the result of the research dissertation of Mr. Morteza Habibi Moghadam, MA student, in critical care nursing that has been conducted after obtain the permission from the Vice Chancellor of Research of Ahvaz Jundishapur University of Medical Sciences and the Ethics Committee. Therefore, the authors would like to appreciate all the professors and loved ones who have collaborated in this way as well as the staff and the authorities of ICU wards of Dr. Ganjavian Hospital, Dezful, for their cooperation in the project.

\section{AUTHORS' CONTRIBUTIONS}

Morteza Habibi Moghaddam contributed as an executor and at the preparing the manuscript, Marzieh Asadi Zakir as supervisor and corresponding author, Dr. Simin Jahani, as Scientific Advisor, Dr. Elham Maraghi as statistical Consultant and Dr. Hakimeh Saadatifar and Dr. Farhad Nanaei contributed as design Collaborator.

\section{CONFLICTS OF INTERESTS}

There are no conflicts of interests in this study.

\section{REFERENCES}

1. Karande GY, Hedgire SS, Sanchez Y, Baliyan V, Mishra V, Ganguli S, et al. Advanced imaging in acute and chronic deep vein thrombosis. Cardiovasc Diagn Ther 2016;6:493-507.

2. Minet C, Potton L, Bonadona A, Hamidfar-Roy R, Somohano CA, Lugosi M, et al. Venous thromboembolism in the ICU: Main characteristics, diagnosis and thromboprophylaxis. Crit Care 2015;19:287.

3. Shakur P, Trzmny M, Fateh H, Farhang S. The frequency of deep vein thrombosis by psonography duplex and related factors in patients hospitalization in intensive care unit. Urmia Med J 2008;19:208-14.

4. Safavi E, Anaraki MR, Sh F, Fard MN. The study of diagnosed venous thromboembolism. Tanaffos 2003;2:15-22.

5. Rezaei HH, Fdaei M, Samadi S, Karakmandi M, Mohammadi FA, Pur SG, et al. Evaluation of risk factors for deep vein thrombosis in patients in surgical intensive units in hospitals of Kerman University of Medical Sciences in 2014. J Iran Soc Anaesthesiol Intensive Care 2015;3:152-8.

6. El-Rashedy NA, Reda NA, Mehany MM. Effect of implementing nursing guidelines on occurrence of deep venous thrombosis for critically Ill patients. J High Inst Public Health 2016;38:200-28.

7. Geerts W, Cook D, Selby R, Etchells E. Venous thromboembolism and its prevention in critical care. J Crit Care 2002;17:95-104.

8. Collins R, MacLellan L, Gibbs H, MacLellan D, Fletcher J. Venous thromboembolism prophylaxis: The role of the nurse in changing practice and saving lives. Aust J Adv Nurs 2010;27:83.

9. Wilbur J, Shian B. Diagnosis of deep venous thrombosis and pulmonary embolism. Am Fam Phys 2012;86:913-9.

10. Mazzolai L, Aboyans V, Ageno W, Agnelli G, Alatri A, Bauersachs R, et al. Diagnosis and management of acute deep vein thrombosis: A joint consensus document from the European society of cardiology working groups of aorta and peripheral circulation and pulmonary circulation and right ventricular function. Eur Heart J 2017.

11. Moheimani F, Jackson DE. Venous thromboembolism: Classification, risk factors, diagnosis, and management. ISRN Hematol 2011; 2011:124610.

12. Cui Y, Wu SH, Sun JH. The investigation of clinical nurse in acknowledge and prevention of deep vein thrombosis. Nurs Res 2009;26:2374e6

13. Squizzato A, Micieli E, Galli M, Gibson N, Gerdes VE, Dentali F, et al. Diagnosis and management of venous thromboembolism: Results of a survey on current clinical practice. Thromb Res 2010;125:134-6.

14. Qiu T, Guo SG, Fang W, Su HB, Chen KR, Zhang K. The high risk categories of deep vein thrombosis in lower extremity. Chin Med J 2013;93:2279e82.

15. Farley A, McLafferty E, Hendry C. Pulmonary embolism: Identification, clinical features and management. Nurs Stand 2009;23:49-56.

16. Mozafar M, Shahabodin MA, Lotfollahzadeh S, Motamedi MA, Sobhiyeh MR. Application of wells criteria, in combination with serum D-dimer to rule out deep vein thrombosis in lower extremities. Scimetr 2013;2:e14770.

17. Hekmatnia A, Pourmoghaddas A. comparison of the results of color doppler sonography and venography in deep vein thrombosis diagnosis. J Qazvin Univ Med Sci 2002;6:51-6.

18. Khavaninzadeh M, Vaeezi M, Saber S, Nik ER, Gharebagh KR. Determination of the frequency of prophylaxis usage against deep-vein thrombosis in hospitalized patients admitted in an educational hospital. Razi J Med Sci 2013;19:77-82.

19. Kazemi T, Qasemi M, Taghavi-shawazi M. Epidemiologic study of patients with DVT in birjand vali-e-asr hospital-(2009-2014) \&58; Short communication. J Birjand Univ Med Sci 2016;23:86-91.

20. Janice L, Hynkel, Kerry H. Brunner and Suddarth's Textbook of Medical-Surgical Nursing, Cardiology. $30^{\text {th }}$ ed. Translation: Mofrad MN, Amini H, Shahri MM, Aliha JM. Washington, DC: American College of Cardiology; 2014. p. 289-96.

21. Hitos K, Cannon M, Cannon S, Garth S, Fletcher JP. Effect of leg exercises on popliteal venous blood flow during prolonged immobility of seated subjects: Implications for prevention of travel-related deep vein thrombosis. J Thromb Haemost 2007;5:1890-5.

22. Habibzadeh H, Rezaeipour N, Safari B, Mohammadi A. The effect of care plan based on nutrition and exercise on deep vein thrombosis in patients undergoing lower limb orthopedic surgery in Urmia imam 
Khomeini hospital in 1391. J Urmi Nurs Midwifery Fac 2013;11:680-7.

23. Schwarz T, Siegert G, Oettler W, Halbritter K, Beyer J, Frommhold R, et al. Venous thrombosis after long-haul flights. Arch Intern Med 2003; 163:2759-64

24. Kwon OY, Jung DY, Kim Y, Cho SH, Yi CH. Effects of ankle exercise combined with deep breathing on blood flow velocity in the femoral vein. Aust J Physiother 2003;49:253-8.

25. Memariyan R, Mohammadi A, Diyanati M. Effects non-pharmacological prophylaxis of deep vein thrombosis in neurosurgical patients. Shahid Sadoghie Med Sci Yazd 2002;10:3-9.

26. Modi S, Deisler R, Gozel K, Reicks P, Irwin E, Brunsvold M, et al. Wells criteria for DVT is a reliable clinical tool to assess the risk of deep venous thrombosis in trauma patients. World J Emerg Surg 2016;11:24. 27. Dybowska M, Tomkowski WZ, Kuca P, Ubysz R, Jóźwik A,
Chmielewski D, et al. Analysis of the accuracy of the wells scale in assessing the probability of lower limb deep vein thrombosis in primary care patients practice. Thromb J 2015;13:18.

28. Silveira PC, Ip IK, Goldhaber SZ, Piazza G, Benson CB, Khorasani R, et al. Performance of wells score for deep vein thrombosis in the inpatient setting. JAMA Intern Med 2015;175:1112-7.

29. Bosson JL, Barro C, Satger B, Carpentier PH, Polack B, Pernod G. Quantitative high D-dimer value is predictive of pulmonary embolism occurrence independently of clinical score in a well-defined low risk factor population. J Thromb Haemost 2005;3:93-9.

30. Shanmugapriya S, Bhuvaneswari K, Rashmi K. A study on the effect of low molecular weight on potassium homeostasis in patients administered heparin for thromboprophylaxis in a tertiary care hospital. Int J Pharm Pharm Sci 2017;9:85-9. 